\title{
LA ADAPTACIÓN DEL LOCUTOR AL INTERLOCUTOR: DINÁMICA DEL SISTEMA VOCÁLICO DEL ESPAÑOL EN FUNCIÓN DE LAS CARACTERÍSTICAS DE LOS PARTICIPANTES EN LA CONVERSACIÓN
}

\author{
D. Poch, C. Dhainaut, K. Huet y B. Harmegnies \\ Universidad Autónoma de Barcelona \\ Université de Mons-Hainaut \\ Dolors.poch@uab.es
}

\begin{abstract}
Resumen
The paper is focused on the hypothesis that the linguistic behaviour of a speaker would depend on how he/she perceives the linguistic status of a person he/she is speaking to. This hypothesis is particularly interesting to study as an index of the mental representation that a determined group has of another, that is to say, the way a speaker adjusts his or her linguistic behaviour to that of the person he or she is speaking to will reflect how the latter is perceived by the speaker being the subject of our study.

This paper presents an exploratory study of the dynamics of the Spanish vocalic system under the effect of the linguistic status variations of the interlocutor. A single speaker, native speaker of Spanish, was recorded on 3 situations in which the differences in the status of the person spoken to are clearly marked: a conversation with a native speaker of Spanish, a conversation with a non-native speaker of Spanish, and a reading task of isolated words.

The assessment of the system's degree of organization in each speaking style was studied using various indices: the $\delta$ index to assess the variation of the degree of centralization of the vowel system under the speaking style variations, the $\Phi$ index, which allows better discrimination between the three speaking styles than the discriminant analysis, to quantify the ratio of the inter vocalic class variability compared to the intra vocalic class variability in each speaking style. The results show both that significant inter-style acoustical differences do exist, and that they can better be stressed by the use of the $\Phi$ index.
\end{abstract}

\section{Introducción}

Durante las últimas décadas, y en el marco de los trabajos ya clásicos de Linblom (1963) y Lindblom (1990), numerosos autores se han interesado por las variaciones sufridas por la señal vocal bajo la influencia de algunas de las condiciones de producción del habla. Dejando aparte el efecto provocado por variables específicas como el acento, el entorno, la velocidad de elocución, etc. (Koopmans van Beinum, 1992; Den Os, 1985; Nord, 1986; Harmegnies y Poch-Olivé, 1992; Van Bergem, 1993), el contexto global de la comunicación ha sido considerado también como una fuente importante de variación de las características acústicas de la señal sonora. Así, en este sentido, se han realizado numerosas investigaciones 
cuyo objetivo era poner de manifiesto la variabilidad de los sonidos del habla en función de las variaciones de estilo determinadas por algunos cambios en la situación de comunicación. Se ha estudiado, por ejemplo, la forma en que cambian las características de los mensajes emitidos en función de las emociones experimentadas por el locutor siendo numerosos los trabajos que se interesan por los estados de ánimo del mismo: depresión, enfermedades diversas, etc. (Wray, 1992; Ball y Duckworth, 1996; Müller, 2000). Se ha estudiado también de qué forma varía el habla cuando el canal de comunicación se ve afectado de alguna manera: las publicaciones que reflejan las investigaciones realizadas sobre el habla en condiciones adversas aportan importantes datos sobre esta cuestión (Asahima, 1994; Matschke, 1994; Payton, Uchanski y Braida, 1994; Neddleman y Crandell, 1995). Abundan igualmente los trabajos que toman en consideración las modificaciones del referente siendo buena muestra de ello los estudios centrados en el lenguaje especializado (Schröder, 1991). El análisis de los fenómenos de "code-switching" (Eastman, 1992; Milroy y Muysken, 1995; Auer, 1998) y de otros fenómenos relacionados con la Pragmática pone de relieve también que las relaciones entre emisor y receptor constituyen uno de los factores de variación de la señal sonora. Hay que señalar, finalmente, que desde el campo de la Sociolingüística se han realizado también importantes estudios sobre la variación, muy especialmente por parte de W. Labov cuyos trabajos constituyen ya un referente clásico (Labov, 1972, 1994, 2001; Eckert, 2000; Docherty, 2003).

Algunos autores, en un intento de sistematización de todas estas líneas de trabajo, han reflexionado sobre la noción misma de estilo de habla y han tratado de definirla, de dar cuenta de los factores que inciden sobre los cambios de estilo y de elaborar una clasificación de estilos. Es especialmente interesante en este sentido el trabajo de M. Eskenazi (1993).

A pesar de que el estudio de los estilos de habla abarca un amplio espectro de perspectivas hay que señalar, no obstante, que muy raramente se ha estudiado la influencia que el estatus del interlocutor puede ejercer sobre las emisiones del locutor. Los trabajos de Labov, los más cercanos a este punto de vista, consideran las características de las producciones del locutor en función de variaciones de las características del propio locutor (edad, sexo, estatus, etc.) pero no en función de variaciones de las características del interlocutor. Un primer trabajo de este tipo fue realizado en 1994 por Harmegnies y Poch y consistía en el análisis de las variaciones sufridas por las vocales del francés cuando un mismo locutor era enfrentado a situaciones en las que debía dirigirse a personas distintas. Dicho estudio puso de manifiesto que el estatus o las características del interlocutor constituyen un factor de variación no menos importante que las características del propio locutor. En este trabajo nos proponemos continuar dicha línea de investigación y analizar las modificaciones sufridas en el habla de un mismo locutor cuando dialoga con un hablante de su propia lengua y cuando dialoga con un hablante para quien el español es lengua extranjera. La percepción del interlocutor debería, en principio, cambiar el comportamiento lingüístico del locutor puesto que no es lo mismo tratar de hacerse comprender por un hablante de nuestra propia lengua que por un hablante de lengua extranjera. Por otra parte, esta perspectiva de estudio es especialmente interesante por cuanto incide en la representación mental que un determinado grupo tiene de otro, es decir, la adaptación del comportamiento lingüístico del locutor a las características del interlocutor reflejará de qué forma es visto este último por el locutor objeto de nuestro estudio. Es ésta una dimensión que la Sociolingüística ha explorado en muy contadas ocasiones y vale, la pena, por tanto profundizar en ella por el aspecto novedoso 
de la misma. Para ello, hemos colocado a un locutor hispanohablante en tres situaciones de comunicación correspondientes a estilos de habla claramente diferenciados en los que es determinante el estatus del interlocutor:

1) Una conversación espontánea con un interlocutor hispanohablante (habla espontánea).

2) Una conversación espontánea (en español) con una interlocutora francófona que estudia español desde hace poco tiempo (habla espontánea intermedia).

3) Lectura de una lista de palabras (habla de laboratorio).

Si nuestra hipótesis es cierta, los resultados mostrarán una diferencia entre las vocales realizadas en la conversación espontánea y las realizadas en la conversación espontánea intermedia. Ello reflejaría que la locutora adapta su forma de hablar a la representación que tiene en su mente del nivel de comprensión de un interlocutor y, en su producción de los sonidos del español, desarrollaría una serie de estrategias cuyo objetivo sería ser comprendida por el hablante no nativo. Y probablemente dichas estrategias serían diferentes de las utilizadas en la situación de habla de laboratorio, situación bien conocida y estudiada en el marco de los trabajos sobre estilos de habla.

Finalmente, nos proponemos explorar otra dimensión de los estudios sobre variación relacionada, en este caso, con los instrumentos empleados en la cuantificación de las variaciones. Normalmente, las herramientas de cuantificación utilizadas para analizar las diferencias entre las distintas situaciones han sido las más usuales de entre las técnicas estadísticas. Esta observación es fundamental puesto que, en cualquier experimento que se lleve a cabo, tan importante es su diseño como los instrumentos que se utilizan para efectuar el análisis, de modo que puede darse el caso de que determinada herramienta de cuantificación no permita poner de manifiesto un fenómeno que puede, en cambio, ser perfectamente estudiado empleando otro instrumento de medición. En este sentido, nos proponemos evaluar el poder discriminante del índice estadístico desarrollado recientemente (Huet y Harmegnies, 2000). A tal fin, y después de realizar el análisis acústico que se describe más adelante, compararemos los resultados obtenidos mediante las técnicas de cálculo más tradicionales con los obtenidos mediante dicho índice. En esta etapa del trabajo, nuestro objetivo será determinar si, puede ser considerado una herramienta de cuantificación relevante que permita poner de manifiesto diferencias entre los estilos de habla que otros instrumentos de cálculo no son capaces de subrayar.

\section{Procedimiento experimental}

\subsection{Corpus y grabaciones}

El corpus se ha obtenido a partir de las producciones vocales de una hablante cuya lengua materna es el español sometida, como ya hemos mencionado, a tres situaciones de comunicación diferentes en función de las características de su interlocutor: una conversación espontánea con un hablante nativo de español ("habla espontánea"); una conversación espontánea con una hablante cuya lengua materna es el francés y que, desde hace pocos meses, está estudiando español en España ("habla espontánea intermedia") y una lectura de pa- 
labras aisladas ("habla de laboratorio"). La locutora cuyas producciones constituyen nuestro objeto de estudio tiene 20 años y estudia Filología Española en la Universidad Autónoma de Barcelona. Los interlocutores son también estudiantes en dicha universidad y, naturalmente, la interlocutora no nativa es una alumna francófona inscrita en el programa Erasmus.

El primer paso del experimento consistió en la grabación de las producciones vocales de la hablante de español nativa durante su conversación espontánea con un interlocutor español. La segunda etapa consistió en la grabación de una conversación entre la hablante de español nativa y la hablante no nativa. En ambas conversaciones se tocaron los mismos temas (situación familiar, estilo de vida, pasatiempos, etc.) de forma que se emplearan las mismas palabras.

Se realizó a continuación la transcripción ortográfica de las dos conversaciones con el fin de obtener una lista común de palabras que pudieran ser utilizadas en la tercera situación. Posteriormente, se pidió al hablante nativo que leyera dicha lista de palabras en la que se habían introducido, además, algunos distractores. Así se obtuvieron, para cada una de las vocales del español (/i e a o u/) 28 palabras extraídas de las dos situaciones de comunicación espontánea y leídas en la situación de laboratorio.

La duración de las conversaciones espontáneas es de, aproximadamente, 50 minutos, ambas grabaciones se realizaron la misma semana y la lectura de palabras de habla de laboratorio se realizó tres semanas después de las otras sesiones.

En total, pues, se han analizado 520 producciones: 28 palabras para cada una de las cinco vocales del español emitidas en tres situaciones de comunicación. Con este procedimiento se ha obtenido una muestra de vocales producida en el mismo entorno y con la misma acentuación para cada uno de los estilos de habla tomados en consideración, siendo ésta, pues, la variable dependiente.

Las grabaciones se han realizado en la cámara insonorizada del Laboratorio de Fonética de la Universidad Autónoma de Barcelona utilizando un micrófono Tascam, un magnetófono de la misma marca modelo 112 y una tabla de mezclas también Tascam modelo 106.

\subsection{Análisis acústico}

El análisis acústico se ha realizado utilizando el programa Phonedit (Phonedit Multimédia. Copyright 1994-1998 SQLAB, BP 88) y ha consistido en la determinación, sobre espectrogramas de banda ancha, de las frecuencias centrales de los dos primeros formantes en el centro de cada vocal y para cada uno de los tres estilos de habla. Se ha realizado así un total de 1.040 mediciones: 2 formantes para cada una de las cinco vocales en cada una de las situaciones de comunicación.

Los valores de frecuencia en herzios ( $\mathrm{f}$ ) se han convertido después en mels (m) utilizando la fórmula de Van Bergem (1993):

$$
\mathrm{m}=2595 \log (1+\mathrm{f} / 700)
$$

\subsection{Resultados}

Una vez obtenidos los valores de las frecuencias de los formantes y los de su posterior conversión en mels hemos procedido al análisis estadístico de los mismos. Para ello y, como 
ya hemos mencionado, hemos utilizado tres herramientas distintas de cuantificación: el índice $\delta$, el análisis discriminante y el índice $\Phi$. Presentamos a continuación los resultados de la aplicación de dichas técnicas.

\subsection{El índice $\delta$}

El índice $\delta$ ha sido desarrollado por Harmegnies y Poch (1992) con el objetivo de analizar la variación del grado de centralización del sistema vocálico en función de la variación del estilo de habla.

Dicho índice se basa en el cálculo de la diferencia entre las distancias euclidianas que expresan el desplazamiento de las distintas realizaciones en el espacio formántico con respecto a un teórico centro (schwa) cuyos formantes primero y segundo se situarían, respectivamente, en $500 \mathrm{~Hz}$ y $1.500 \mathrm{~Hz}$.

El índice se define como:

$$
\delta=\left[\left(F_{1}-500\right)^{2}+\left(F_{2}-1500\right)^{2}\right]^{1 / 2}-\left[\left(f_{1}-500\right)^{2}+\left(f_{2}-1500\right)^{2}\right]^{1 / 2}
$$

donde:

- F1 y F2 son los valores del primero y segundo formantes de una vocal en un determinado estilo de habla;

- $\mathrm{fl}$ y $\mathrm{f} 2$ son los valores del primero y segundo formantes de una vocal en otro estilo de habla;

- 500 y 1500 son los valores del primer y segundo formantes de schwa (en hercios).

Así, $\delta$ proporciona, para cada par de vocales, los valores de las diferencias de las distancias euclidianas entre dos estilos de habla. Los valores más elevados del índice indican que la diferencia entre las vocales consideradas es grande mientras que ocurre al revés cuando los valores del índice son más bajos. Una vez obtenidos los valores de dichas diferencias lo interesante es, obviamente, evaluar mediante tests estadísticos el grado de significación de las diferencias inter-estilos. Este ha sido el procedimiento de cálculo que hemos aplicado en primer lugar y presentamos a continuación los valores de $\delta$ así como los valores de significación de las variaciones proporcionados por los tests estadísticos. Cuanto más cercanos a 0 están los valores obtenidos para " $p$ " menor es el riesgo de error al afirmar que existen diferencias significativas. Hemos utilizado el t-test de Student así como un test no paramétrico, el t-test de Wilcoxon y hemos calculado tres índices $\delta$ :

1. $\delta$ 1: habla de laboratorio y habla espontánea intermedia

2. $\delta 2$ : habla de laboratorio y habla espontánea

3. $\delta 3$ : habla espontánea y habla espontánea intermedia 
Tabla 1: $\delta 1$ (habla de laboratorio y habla espontánea intermedia)

\begin{tabular}{|c|c|c|c|c|c|}
\hline Vocal & $\begin{array}{c}\text { Media } \\
(\mathbf{m e l s})\end{array}$ & $\begin{array}{c}\text { Desv. } \\
\text { Estan. (mels) }\end{array}$ & $\mathbf{N}$ & Student t: $\mathbf{p}$ & Wilcoxon T: $\mathbf{p}$ \\
\hline$/ \mathrm{i} /$ & 57.02 & 45.99 & 28 & .000 & .000 \\
\hline$/ \mathrm{e} /$ & 51.45 & 68.11 & 28 & .000 & .002 \\
\hline $\mathrm{/a} /$ & 64.77 & 63.78 & 28 & .000 & .000 \\
\hline$/ \mathrm{d} /$ & -94.16 & 99.77 & 28 & .000 & .000 \\
\hline$/ \mathrm{u} /$ & -41.51 & 138.52 & 28 & .124 & .122 \\
\hline
\end{tabular}

Tabla 2: $\delta 2$ (habla de laboratorio y habla espontánea)

\begin{tabular}{|c|c|c|c|c|c|}
\hline Vocal & $\begin{array}{c}\text { Media } \\
(\mathbf{m e l s})\end{array}$ & $\begin{array}{c}\text { Desv. } \\
\text { Estan. (mels) }\end{array}$ & $\mathbf{N}$ & Student t: $\mathbf{p}$ & Wilcoxon T: $\mathbf{p}$ \\
\hline$/ \mathrm{i} /$ & 112.35 & 92.86 & 28 & .000 & .000 \\
\hline$/ \mathrm{e} /$ & 87.18 & 66.93 & 28 & .000 & .000 \\
\hline$/ \mathrm{a} /$ & 69.83 & 92.42 & 28 & .000 & .001 \\
\hline$/ \mathrm{o} /$ & 151.42 & 136.81 & 28 & .000 & .000 \\
\hline$/ \mathrm{u} /$ & 116.15 & 141.80 & 28 & .000 & .001 \\
\hline
\end{tabular}

Tabla 3: $\delta 3$ (habla espontánea y habla espontánea intermedia)

\begin{tabular}{|c|c|c|c|c|c|}
\hline Vocal & $\begin{array}{c}\text { Media } \\
\text { (mels) }\end{array}$ & $\begin{array}{c}\text { Desv. } \\
\text { Estan. (mels) }\end{array}$ & $\mathbf{N}$ & Student t: $\mathbf{p}$ & Wilcoxon T: $\mathbf{p}$ \\
\hline$/ \mathrm{i} /$ & 55.33 & 85.62 & 28 & .002 & .005 \\
\hline$/ \mathrm{e} /$ & 35.73 & 81.28 & 28 & .028 & .034 \\
\hline$/ \mathrm{a} /$ & 5.06 & 83.21 & 28 & .750 & .946 \\
\hline$/ \mathrm{o} /$ & -57.27 & 76.17 & 28 & .000 & .001 \\
\hline $\mathrm{h} /$ & -74.63 & 96.49 & 28 & .000 & .000 \\
\hline
\end{tabular}

Los resultados más claros son los que figuran en la Tabla 2 en la que se evalúan las diferencias entre habla de laboratorio y habla espontánea pues las diferencias son claramente significativas para todas las vocales. Ello está en consonancia con los resultados obtenidos en otros trabajos ya citados en los que se comparan los mismos estilos de habla. Los resultados indicados en las Tablas 1 y 3 , en cambio, no son tan claros: cuando se comparan el habla de laboratorio y el habla espontánea con el habla espontánea intermedia las diferencias aparecen a veces como significativas y, en otras ocasiones, como no significativas. Una posible interpretación de estos datos consistiría en pensar que, efectivamente, no existen grandes diferencias entre dichos estilos de habla con lo cual la hipótesis de la adaptación 
al locutor quedaría invalidada. No obstante, otra forma posible de ver las cosas consiste en preguntarse si los resultados serían diferentes si se utilizara otra técnica de medición tal como mencionábamos en la introducción.

\subsection{Análisis discriminante}

Hemos efectuado, pues, una segunda aproximación al problema mediante la técnica del análisis discriminante. Así, para cada situación de comunicación, los fonemas han sido considerados como categorías a priori y las frecuencias de los formantes (F1 y F2) de las realizaciones del corpus, como variables discriminantes. Una vez obtenidas las funciones discriminantes, éstas han sido utilizadas para simular una tarea de reconocimiento de las distintas realizaciones en cada una de las situaciones de comunicación de forma que cada producción vocálica fuera reconocida como perteneciente a una de las cinco categorías fonémicas del español.

Los resultados obtenidos se presentan a continuación:

Tabla 4: matriz de confusiones: habla de laboratorio, media: $96.4 \%$

\begin{tabular}{|l|c|c|c|c|c|}
\hline $\begin{array}{l}\text { Vocal } \\
\text { emitida }\end{array}$ & $\begin{array}{c}\text { Vocal } \\
\text { reconocida }\end{array}$ & $\begin{array}{c}\text { Vocal } \\
\text { reconocida }\end{array}$ & $\begin{array}{c}\text { Vocal } \\
\text { reconocida }\end{array}$ & $\begin{array}{c}\text { Vocal } \\
\text { reconocida }\end{array}$ & $\begin{array}{c}\text { Vocal } \\
\text { reconocida }\end{array}$ \\
\hline$[\mathrm{i}]$ & $/ \mathrm{i} /$ & $/ \mathrm{e} /$ & $/ \mathrm{a} /$ & $/ \mathrm{o} /$ & $-\mathrm{u} /$ \\
\hline$[\mathrm{e}]$ & $100 \%$ & - & - & - & - \\
\hline$[\mathrm{a}]$ & $3.6 \%$ & $96.4 \%$ & - & - & - \\
\hline$[\mathrm{o}]$ & - & - & $100 \%$ & $85.7 \%$ & $14.3 \%$ \\
\hline$[\mathrm{u}]$ & - & - & - & - & $100 \%$ \\
\hline
\end{tabular}

Tabla 5: matriz de confusiones: habla espontánea intermedia, media: $82.9 \%$

\begin{tabular}{|l|c|c|c|c|c|}
\hline $\begin{array}{l}\text { Vocal } \\
\text { emitida }\end{array}$ & $\begin{array}{c}\text { Vocal } \\
\text { reconocida }\end{array}$ & $\begin{array}{c}\text { Vocal } \\
\text { reconocida }\end{array}$ & $\begin{array}{c}\text { Vocal } \\
\text { reconocida }\end{array}$ & $\begin{array}{c}\text { Vocal } \\
\text { reconocida }\end{array}$ & $\begin{array}{c}\text { Vocal } \\
\text { reconocida }\end{array}$ \\
\hline & $/ \mathrm{i} /$ & $/ \mathrm{e} /$ & $/ \mathrm{a} /$ & $/ \mathrm{o} /$ & $/ \mathrm{u} /$ \\
\hline$[\mathrm{i}]$ & $92.9 \%$ & $7.1 \%$ & - & - & - \\
\hline$[\mathrm{e}]$ & $10.7 \%$ & $85.7 \%$ & $3.6 \%$ & - & - \\
\hline$[\mathrm{a}]$ & - & $3.6 \%$ & $96.4 \%$ & - & - \\
\hline$[\mathrm{o}]$ & - & - & $7.1 \%$ & $60.7 \%$ & $32.1 \%$ \\
\hline$[\mathrm{u}]$ & - & - & - & $21.4 \%$ & $78.6 \%$ \\
\hline
\end{tabular}


Tabla 6: matriz de confusiones: habla espontánea, media: $82.1 \%$

\begin{tabular}{|l|c|c|c|c|c|}
\hline $\begin{array}{l}\text { Vocal } \\
\text { emitida }\end{array}$ & $\begin{array}{c}\text { Vocal } \\
\text { reconocida }\end{array}$ & $\begin{array}{c}\text { Vocal } \\
\text { reconocida }\end{array}$ & $\begin{array}{c}\text { Vocal } \\
\text { reconocida }\end{array}$ & $\begin{array}{c}\text { Vocal } \\
\text { reconocida }\end{array}$ & $\begin{array}{c}\text { Vocal } \\
\text { reconocida }\end{array}$ \\
\hline & $/ \mathrm{i} /$ & $/ \mathrm{e} /$ & $/ \mathrm{a} /$ & $/ \mathrm{o} /$ & $/ \mathrm{u} /$ \\
\hline$[\mathrm{i}]$ & $85.7 \%$ & $14.3 \%$ & - & - & - \\
\hline$[\mathrm{e}]$ & $14.3 \%$ & $85.7 \%$ & - & - & - \\
\hline$[\mathrm{a}]$ & - & $3.6 \%$ & $89.3 \%$ & $7.1 \%$ & - \\
\hline$[\mathrm{o}]$ & - & - & $7.1 \%$ & $71.4 \%$ & $21.4 \%$ \\
\hline$[\mathrm{u}]$ & - & - & - & $21.4 \%$ & $78.6 \%$ \\
\hline
\end{tabular}

Como puede verse en la Tabla 4, mediante esta técnica se obtiene una excelente discriminación en habla de laboratorio: se reconocen correctamente un $96,4 \%$ de las realizaciones. Dichos resultados coinciden con los de otros trabajos y es necesario también recordar que, en habla de laboratorio, el espacio vocálico está claramente estructurado y las características acústicas de los sonidos realizados son muy diferentes para cada categoría vocálica. En cambio, en habla espontánea, como puede verse en la Tabla 6, el reconocimiento correcto alcanza tan sólo al $82,1 \%$ de las realizaciones analizadas lo cual coincide igualmente con los valores obtenidos en trabajos anteriores. En habla espontánea el espacio vocálico está menos estructurado que en habla de laboratorio y las características acústicas de los sonidos no están tan diferenciadas como lo están las del habla de laboratorio, de ahí que el porcentaje de reconocimiento correcto en el análisis discriminante sea claramente menor que en habla de laboratorio. Así pues, comparando ambos resultados puede afirmarse que el habla de laboratorio y el habla espontánea son sustancialmente diferentes.

La Tabla 5 indica que el habla espontánea intermedia, con un porcentaje del $82,9 \%$ de reconocimiento correcto, se sitúa muy cerca de las características acústicas que presentan los sonidos en situación de habla espontánea. Estos datos nos sitúan de nuevo ante el dilema de considerar que las realizaciones pertenecientes al habla espontánea intermedia y las pertenecientes al habla espontánea son muy similares o bien estimar que sería necesario abordar la cuestión mediante otra técnica de cuantificación que tal vez fuera capaz de diferenciar las tres situaciones de comunicación.

\subsection{El índice $\Phi$}

Así lo hemos hecho utilizando en este caso el índice $\Phi$ (Huet y Harmegnies, 2000; Huet, Harmegines y Poch-Olivé, 2001). Dicho índice puede caracterizarse como una forma de medir globalmente la diferenciación. Da cuenta, mediante una única cifra, del grado de contraste existente entre las realizaciones de los fonemas en el espacio vocálico de un locutor determinado para una situación de comunicación específica.

Igual que otros índices de este tipo, el índice $\Phi$ se basa en una concepción topográfica del sistema vocálico: las realizaciones son consideradas puntos de un plano cuyos ejes son 
las frecuencias del primer y segundo formantes. Dado que las diferentes actualizaciones de una vocal determinada constituyen realizaciones de un mismo fonema, es esperable que ocupen una zona determinada del plano vocálico. Si el locutor realizada todas las actualizaciones de un fonema vocálico de manera idéntica, todos los puntos (= vocales) se confundirían y la región considerada del plano vocálico tendría una superficie infinitamente pequeña. En la realidad, las manifestaciones acústicas producidas por los locutores varian y aparece, pues, una cierta dispersión alrededor de un punto virtual (considerado como la realización canónica del fonema por parte del locutor en la situación considerada).

Es sabido, por otra parte, que para un fonema vocálico determinado, la dispersión alrededor del punto canónico puede ser más o menos importante en función del estilo de habla. $Y$ es sabido también que las áreas de dispersión de las vocales pueden cambiar de posición en el espacio vocálico en función del estilo de habla. El índice $\delta$, como hemos visto, es sensible a una de estas formas de cambiar de posición: la que está relacionada con la centralización.

El índice $\Phi$ pretende evaluar el grado de diferenciación del sistema basándose en la relación entre la variabilidad intra-áreas de dispersión y la variabilidad inter-áreas de dispersión. En este sentido, el índice $\Phi$ puede ser considerado una generalización, en un espacio bidimensional, de lo que sería un análisis de varianza univariado realizado en un espacio unidimensional.

Para calcular el índice $\Phi$ es necesario, en primer lugar, determinar en el interior de cada área de dispersión las distancias entre las realizaciones observadas y la realización media (varianza intra-áreas). La combinación de estas distancias proporciona una estimación de la dispersión intra-áreas. A continuación hay que efectuar una estimación del valor de las distancias que separan las áreas entre sí. El valor así obtenido (varianza inter-áreas) puede ser relacionado con los valores de varianza intra-áreas lo cual proporciona una valores que dan cuenta del grado de diferenciación del sistema: el índice $\Phi$.

Si la dispersión en el interior de cada área es débil, el denominador de la relación es pequeño y el valor obtenido es grande; si las áreas están muy separadas unas de otras, el numerador es grande y el valor obtenido es también elevado. $\mathrm{Si}$, en un caso extremo, las realizaciones se presentaran totalmente indiferenciadas y si las áreas se superpusieran completamente, la variabilidad intra-áreas sería idéntica a la variabilidad inter-áreas. En ese caso, el valor de las varianzas sería igual a 1 .

Con respecto al índice $\delta$, el índice presenta la ventaja de proporcionar una medida que caracteriza en estado determinado del sistema, mientras que $\delta$ puede expresar únicamente la comparación de dos estados (habla de laboratorio y habla espontánea, habla espontánea y habla espontánea intermedia, etc.). Además, si bien $\delta$ es sensible a los movimientos de las áreas de dispersión lo es solamente a los desplazamientos que implican centralización mientras que $\Phi$, en cambio, es sensible a cualquier tipo de movimiento y no necesita de la formulación de una hipótesis a priori como la de la existencia de una vocal neutra caracterizada por valores teóricos.

Igual que la técnica basada en el análisis discriminante, el índice $\Phi$ permite efectuar una estimación del grado de organización del sistema. No obstante, proporciona una alternativa de medición al proceso estrictamente dicotómico sobre el que reposa el cálculo del porcentaje final de reconocimiento correcto puesto que este último se basa en la decisión binaria de pertenencia o no de un elemento a un conjunto. Se trata, pues, de un análisis mucho más 
fino puesto que se basa en la apreciación de grados de diferencia mientras que el análisis discriminante se basa en decisiones de carácter estrictamente dicotómico.

El cálculo del índice para las vocales objeto de estudio ha proporcionado los siguientes resultados:

- Habla de laboratorio: 491,75

- Habla espontánea intermedia: 306,29

- Habla espontánea: 212,80

Estos valores pueden representarse tal como aparecen en la figura 1:

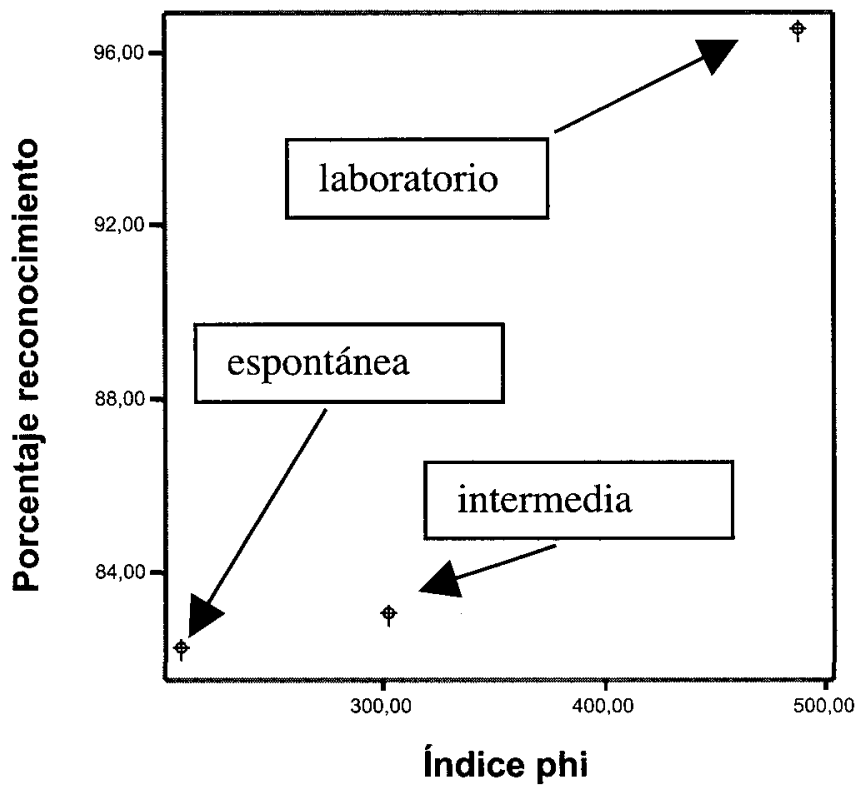

Figura 1: resultados del análisis discriminante y del procedimiento de análisis mediante la aplicación del índice

Como puede verse, este índice, contrariamente a los otros dos procedimientos de cuantificación, permite establecer una clara diferencia entre los tres estilos de habla considerados en este trabajo pues distingue tres grupos de valores, cada uno de ellos correspondiente a una situación diferente de producción del habla.

Estos resultados permiten confirmar nuevamente la hipótesis, ya verificada en estudios anteriores, de que el sistema vocálico varía en función del estilo de habla pudiendo establecerse diferencias no sólo entre el habla de laboratorio y el habla espontánea sino también entre el habla espontánea y el habla espontánea intermedia. Por otra parte, queda también confirmada la hipótesis de que las técnicas utilizadas para cuantificar los fenómenos tienen un papel fundamental en el estudio de los mismos pues no todas son capaces de poner de manifiesto los distintos comportamientos de las realizaciones de los sonidos del habla. 


\section{Conclusiones}

Las conclusiones que pueden extraerse de este estudio son de orden diferente: por una parte, algunas de ellas están relacionadas, como ya hemos mencionado, con cuestiones de carácter metodológico ligadas a la cuantificación de los fenómenos. En ese sentido, los resultados obtenidos permiten afirmar que el índice es el único de los tres procedimientos utilizados que ha permitido mostrar claramente una diferencia entre los tres estilos de habla considerados en este estudio lo cual implica que una posible vía de estudio de los fenómenos de variación radica en las técnicas numéricas derivadas del análisis de varianza.

Por otra parte, y en el marco de una reflexión de carácter mucho más general, gracias a dichos cálculos, ha aparecido claramente en la dinámica del sistema vocálico del español, una situación intermedia entre el habla de laboratorio y el habla espontánea. Ello nos remite a la primera de nuestras hipótesis: el locutor se adapta, en su modo de hablar, al interlocutor a partir de la representación que posee de las posibilidades de comprensión de la lengua por parte de éste. Los resultados obtenidos para el habla espontánea intermedia pueden explicarse en el marco del modelo H \& H de Lindblom (1990). La locutora tiene a su disposición una serie de aspectos contextuales, pragmáticos y semánticos que se suman a la señal vocal en tanto vectores portadores de información para el receptor y los utiliza para hacerse comprender de tal forma que sus producciones, con respecto al habla de laboratorio, presentan un cierto grado de desorganización. Pero dicha desorganización es menor en relación con la observada en habla espontánea y ello es debido a que, en habla espontánea intermedia, la informante, al ser consciente de que está hablando con un nonativo, se esfuerza por transmitir la mayor cantidad posible de información a través de la señal de forma que el grado de desorganización del sistema no es nunca tan elevado como el de habla espontánea. Este comportamiento subraya, como apuntábamos al principio de este trabajo, que uno de los factores de variación en el habla de un locutor determinado está relacionado con un factor totalmente ajeno a dicho locutor: el interlocutor, la persona con quien está hablando. Así, a la vez que confirman nuestra hipótesis de partida, estos resultados muestran un camino que debe ser explorado con mayor profundidad para llegar a comprender qué influencia tienen sobre la producción los distintos factores que intervienen en la situación de comunicación incluyendo en ellos las características del interlocutor a las que el locutor / emisor debe adaptarse para que la comunicación funcione de manera eficaz.

\section{Referencias bibliográficas}

Asahina, N. (1994): "A study of speech intelligibility in traffic noise", JORLJ, 97, 3, págs. 401-413. Auer, P. (ed.) (1998): Code-Switching in Conversation: Language, Interaction and Identity. London, Peter Lang.

Ball, M. y M. Duckworth (eds.) (2000): Pragmatics in Speech and Language Patology: Studies in clininal application. The Hague, John Benjamins.

Van Bergem, D. R. (1993): "Acoustic vowel reduction as a function of sentence accent, word stress, and word class", Speech Communication, 12, págs. 1-23.

Den Os, E. (1985): "Perception of speech rate: a scaling experiment", Progress Report Institute of Phonetics. University of Utrecht, 13, págs. 299-308. 
Docherty, G. J. (2003): "Speaker, Community, Identity: Empirical and Theoretical Perspectives on Sociophonetic Variation". En Proceedings of 15 $15^{\text {th }}$ ICPhS, págs. 11-16.

Eastman, C. M. (ed.) (1992): Codeswitching. Clevedon, Multilingual Maters.

Eckert, P. (2000), Linguistic Variation as Social Practice. Oxford, Blackwell.

Eskenazi, M. (1993): "Trends in Speaking Style Research". En Proceedings Eurospeech'93. Berlin, vol. 1, págs. 501-509.

Harmegnies, B. y D. Poch-Olivé (1992): "A study of style-induced vowel variability: laboratory versus spontaneous speech in Spanish", Speech Communication, 11, págs. 429-437.

Harmegnies, B. y D. Poch-Olivé (1994): "Formants frequencies variability in French vowels under the effect of various speaking styles", Journal de Physique, IV, págs. 509-512.

Huet, K. y B. Harmegnies (2000): "Contribution à la quantification du degré d'organisation des systèmes vocaliques”. En Actes des XXIIIèmes Journées d'Étude sur la Parole - JEP'2000, págs. $225-228$.

Huet, K.; Harmegnies, B. y D. Poch-Olivé (2001): "Une méthode statistique pour le contrôle des changements vocaliques sous l'effet du style de parole. Application à l'espagnol", TRANEL, 34/35, págs. 233-249.

Koopmans van Beinum, F. (1992): "The role of focus words in natural and in synthetic continuous speech: acoustic aspects", Speech Communication, 11, págs. 439-450.

Labov, W. (1994): Principles of Linguistic Changes: Internal Factors. Oxford, Blackwell.

Labov, W. (2001): Principles of Linguistic Changes: Social Factors. Oxford, Blackwell.

Lindblom, B. (1963): "Spectrographic study of vowel reduction", JASA, 35, págs. 1773-1781.

Lindblom, B. (1990): "Explaining Phonetic Variation: A Sketch of the H and H Theory". En Hardcastle, W. y A. Marchal (eds.), Speech Production and Speech Modelling. Dordrecht, Kluwer, págs. 403-440.

Matschke, R. G. (1994): "Communication in noise: Speech intelligibility of airplane pilots with and without active noise communication", $H N O$, 42, 8, págs. 499-504.

Milroy, L. y P. Muysken (1995): One Speaker, Two Languages. Cross-disciplinary Perspectives on Code-switching. Cambridge, Cambridge University Press.

Müller, N. (ed.) (1996): Advances in Clinical Phonetics. The Hague, John Benjamins.

Needleman, A. R. y C. C. Crandell (1995): "Speech recognition in noise by hearing-impaired and noise-masked normal-hearing listeners", $J A A A, 6$, págs. 414-424.

Nord, L. (1986): "Acoustic studies of vowel reduction in Swedish", STL-QPSR, 4, págs. 19-36.

Payton, K. L., Uchanski, R. M. y L. D. Braida (1994): "Intelligibility of conversational and clear speech in noise and reverberation for listeners with normal and impraired hearing", $J A S A, 95,3$, págs. 1581-1592.

Schröder, H. (1991): Subject-oriented Texts. Languages for Special Purposes and Text Theory. Berlin, De Gruyter.

Wray, A. (1992): The Focusing Hypothesis: The Theory of left hemisphere lateralised language reexamined. The Hague, John Benjamins. 